received solid organ or bone marrow transplants and take immunosuppressive drugs, a chronic or persistent hepatitis can develop defined by viremia for greater than 3 months. Spontaneous clearance can occur with reduction of immunosuppressive load. Failing this, there is evidence these patients can be successfully treated with ribavirin. The goal is sustained viral response (SVR) with undetectable Hepatitis E RNA in blood and stool for 6 months after treatment. A recent analysis of 69 patients with solid organ transplants reported SVR of 78\%. Some success has also been seen in bone marrow transplants. We aim to present our experience of treating persistent Hepatitis E infection with ribavirin.

Methods Retrospective analysis of clinical records and the pathology database.

Results Following a screening program at the Queen Elizabeth Hospital, Birmingham we have treated 11 transplant recipients with ribavirin over the last 18 months. Transplant types included 4 liver, 3 kidney, 1 heart and kidney combined and 3 bone marrow. The median age of the cohort was 44, patients were a median of 2 years out from transplant and taking a median of 2 immunosuppressants. ALT was raised in all patients, median value 155 (46 to 599). Ribavirin treatment was given for 12 weeks and continued if stool remained RNA positive at week 12; the median course was 14 weeks. Ribavirin was generally well tolerated although one patient had a treatment break at 2 weeks due to diarrhoea and vomiting; 2 needed EPO support. SVR was observed in 10/11 patients $(91 \%)$ of whom $9 / 10(90 \%)$ have continued to show SVR until the present time (mean 2.2 months). ALT responded in all patients, median value 20 (11 to 31 ). One patient did not achieve SVR and relapsed at 3 months; one patient who did achieve SVR relapsed at 10 months. 2 bone marrow recipients had received high dose steroids for graft versus host disease $(\mathrm{GvHD})$ and liver biopsy was undertaken to distinguish Hepatitis E from GvHD: in both cases the biopsy showed a non-specific chronic hepatitis in keeping with Hepatitis E.

Conclusions Our Results show SVR of 91\% with ribavirin in persistent Hepatitis E infection in a diverse cohort with different types of transplant including bone marrow. The fact that all patients had abnormal ALT means that persistent Hepatitis E must be considered as a cause of raised liver enzymes in transplant recipients. Of particular interest is the need to distinguish GvHD from persistent Hepatitis $\mathrm{E}$ in bone marrow recipients as the therapeutic approaches are fundamentally different and increasing doses of steroids, and rituximab in one case, led to worsening viral loads in our cohort. Ongoing follow-up will take place in our cohort and look for late relapse.

\section{ADWE-05 PRE-EMPTIVE TIPSS IMPROVES CLINICAL OUTCOMES IN PATIENTS WITH VARICEAL HAEMORRHAGE: A RETROSPECTIVE COHORT STUDY}

Ihteshamul Haq*, Meagan Lau, Emma Jongman, Robert Crichton, Lalitha Chinnappan, Simon Ollif, Homoyon Mehrzad, Salil Karkhanis Karkhanis, Catherine Snelson, Nick Murphy, Dhiraj Tripathi. Queen Elizabeth Hospital, Birmingham, UK; Introduction

\subsection{6/gutjnl-2018-BSGAbstracts.214}

Variceal haemorrhage is a medical emergency associated with a 30 day mortality of $15 \%$ and a re-bleeding rate of $26 \%$. If there is failure to control bleeding endoscopically salvage TIPSS is reported to control bleeding in $90 \%-100 \%$ of cases, with re-bleeding rates of $6 \%-16 \%$. However mortality remains high. Approximately 20\% of patients are 'high risk'; here early or pre-emptive TIPSS, before treatment failure (up to 5 days post bleed), can significantly reduce morbidity/mortality. A recent multicentre study has shown that pre-emptive covered TIPSS was associated with good outcomes but poses significant challenges in the 'real world' situation. We evaluated the clinical outcomes of patients undergoing a covered TIPSS following an acute variceal bleed and subsequent admission to the Intensive Care Unit with focus on pre-emptive TIPSS strategies.

Method We used the CRIS Radiology Information System to construct a database of all patients undergoing a TIPSS for variceal haemorrhage at between 2013 and 2016. For each patient we collected a wide range of data, which were analysed using SPSS (SPSS Inc. Chicago IL, USA) and expressed as means \pm SD. Mortality data were analysed using the KaplanMeier Method.

Results We studied 96 patients with a mean age 51.0 12.1 years, M:F ratio (67:29), alcohol related liver disease $(59.4 \%)$, MELD score $15.8 \pm 6.8$, and follow up $25.4 \pm 18.3$ months. All TIPSS procedures were successful, and indication was as salvage $(42 \%)$, pre-emptive (44\%), and secondary prevention $(14 \%)$. Initial management involved band ligation in combination with vasoconstrictors and/or Sengstaken- Blakemore Tube in all cases. Patient mortality at 6 weeks, 6 months, 12 months and 24 months was 19\%, 24\%, 30\% and 34\% respectively. MELD score $>15$ was significantly associated with mortality following multivariate analysis. Six week mortality was significantly higher with salvage TIPSS compared to pre-emptive strategy $(33 \%$ versus $9 \%, \mathrm{p}<0.05)$, even after controlling for MELD. There was no difference in mortality between pre-emptive and secondary prevention strategies. The overall re-bleeding rate was $2 \%$.

Conclusion The outcomes of patients admitted to ICU following a variceal bleed are good and comparable to published literature. This is probably a reflection of high-standard ICU care. Pre-emptive TIPSS up to five days following the index variceal bleed Results in significantly better outcomes than salvage TIPSS, with mortality comparable to TIPSS for secondary prevention. However, the currently hub and spoke model of TIPSS services in the UK is unable to accommodate a preemptive strategy in all region

\section{PWE-073 SPLEEN STIFFNESS VIA ACOUSTIC RADIATION FORCE IMPULSE IN HIV PATIENTS WITH NON CIRRHOTIC PORTAL HYPERTENSION}

Sebastiana Atzori*, Lucy Garvey, Tim Hoogenboom, James Maurice, Graham Cooke, Simon Taylor-Robinson. Imperial College, London, UK

\subsection{6/gutjnl-2018-BSGAbstracts.215}

Introduction Several HIV centres describe small cohorts of patients with non-cirrhotic portal hypertension (NCPH). Didanosine (ddI) exposure has been associated and the optimal monitoring and management of these patients remains unclear. Non-invasive assessment of the spleen is now being utilised to assess splenic stiffness in cirrhotic portal hypertension and predict outcomes. This technique has not previously been studied in patients with HIV and NCPH.

Methods Acoustic Radiation Force Impulse (ARFI) elastography was performed using Philips EPIQ7 $7^{\mathrm{TM}}$ to simultaneously assess 